Methods Studies were selected by a systematic search in Medline (PubMed) and BIREME (LILACS, IBECS, BINACIS, BDENF, Index Psicologia, WHOLIS, MedCarib and Coleciona SUS) databases. Inclusion criteria were articles in English, Spanish or Portuguese, providing statistical analyses on risk factors for workplace bullying. Quality was assessed using an adapted version of the Downs and Black checklist. PRISMA and MOOSE guidelines were used for reporting papers.

Results Fifty-one papers were included in the review. $70.6 \%$ were from European countries. Women were reported to be at higher risk of being bullied in most studies. The association of age, marital status and personality traits with bullying varied across studies. Authoritarian and laissez-faire leadership styles were positively associated to bullying. Several occupational risks related to the work organisation and psychosocial factors - such as the stress - were strongly associated to workplace bullying.

Conclusions Findings from this review highlight the central role of organisational factors on bullying determination, in which the human resource management is a key distal factor. Policies to prevent bullying must address the culture of organisations, facing the permanent challenge of developing safe psychosocial work environments.

\section{O2B.3 WORK RELATED STRESS FOR NEW ZEALAND WORKERS IN HIGH RISK SECTORS: WHAT CAN WE LEARN FROM A DEMOGRAPHIC ANALYSIS?}

Kirsten Lovelock, Trang Khieu*. WorSafe New Zealand, Wellington, New Zealand

\subsection{6/OEM-2019-EPI.39}

The physical and psychological outcomes of work related stress place a burden on individuals, their families, workplaces and communities. Work related stress is a health and socio-economic and political problem. It reduces work performance; drives higher rates of absenteeism or sick leave; can increase rates of injury; prompt high staff turnover; and, can prompt poor health behaviours such as excessive drinking or taking of drugs. The research record focussing on work related stress in New Zealand (NZ) is small and uneven, but growing. The aim of this study was to explore the prevalence of work-related stress among workers by a set of demographic characteristics. The study used data from the Health and Safety Attitudes and Behaviour Survey (HSABS) 2016. A total of 2190 workers in the four high risk industries (agriculture, forestry, construction and manufacturing) were interviewed about their perceptions towards work-related stress. Weighting was conducted to control inherent biases. Differences between workers were examined by age, sex, ethnicity, qualifications and migrant factors (e.g. being born in NZ or arriving in NZ within five years). Overall, $11 \%$ of workers responded that they had experienced with work-related stress in the last 12 months. Work-related stress was more statistically prevalent among people from 25 to 34 years of age or those having a bachelor's degree. Also, people being females or Maori or not born in NZ or first arrived in NZ in the last five years were more likely to have work-related stress. Findings from the study could allow better targeted and informed psychosocial health interventions to be implemented at workplace.

\section{O2B.4 FACTORS ON SICKNESS ABSENCE AMONG WORKERS} AND MODERATING EFFECT OF JOB STRESS IN KOREA

${ }^{1}$ Hyang-Woo Ryu*, ${ }^{2}$ Deog-Hwan Moon, ${ }^{3}$ Sang-Gil Lee. ${ }^{1}$ Occupational Safety and Health Research Instutute, KOSHA, UIsan, South Korea; ${ }^{2}$ Department of Public Health, Inje University, Busan, South Korea; ${ }^{3}$ Occupational Safety and Health Research Instutute, KOSHA, Ulsan, South Korea

\subsection{6/OEM-2019-EPI.40}

Objective The purpose of this study was to analyze the various factors affecting the sickness absence of Korean workers and to confirm the moderating effect of job stress.

Method We analyzed the data of nationwide labor environment survey conducted by the Korea Occupational Safety and Health Agency (KOSHA) in 2011. This study covered 49870 workers, excluding abnormal values. We analyzed the effects of the work environment and the moderating effect of job stress to the absence of sickness.

Results The number of sickness absent from the study was 4621 (9.3\%), total sickness absence was 30,640 days, and the average period of sickness absence was 6.6 days. The factor with significant difference of illness absenteeism was education, monthly income, size of workplace, drinking opportunity, worker position, musculoskeletal disease risk factor, and subjective health status. The interaction effect between the control variable and the independent variable was significantly different from the job stress $\times$ musculoskeletal disorder factor.

Sickness absence was higher in middle school graduates, monthly incomes more than one million won, workplaces with 10 or more workers, and in the non-alcoholic group.

Musculoskeletal disorders and subjective health status showed a positive effect on sickness absence. The number of days of sickness absence increased and the subjective health condition got worse as the musculoskeletal disorders increased. As job stress increased, sickness absence increased. We found that the job stress interacted with musculoskeletal disease factors and affected sickness absence.

Conclusion It is necessary to prepare measures to improve subjective health condition, and to fully utilize the moderating job stress factors to reduce the factors of musculoskeletal diseases.

\section{Workers' Compensation/Return to Work}

\section{O2C.1 WORKER COMPENSATION: ARE EPIDEMIOLOGICAL STUDIES FIT FOR PURPOSE?}

Lesley Rushton. Imperial College London, London, UK

\subsection{6/OEM-2019-EPI.41}

Introduction Every year, large numbers of workers have an injury at work or develop a work-related disease. A range of worker compensation systems exist across countries for which epidemiological studies potentially provide critical evidence. This paper discusses the adequacy of current epidemiological research for this purpose.

Methods Compensation schemes need to identify the occupational circumstances, or dose, that increase (e.g. double) the risks of the disease, and to define these so they can be administered effectively by decision-makers who lack epidemiological 